\title{
Human Telomerase Gene and High-Risk Human Papillomavirus Infection are Related to Cervical Intraepithelial Neoplasia
}

\author{
Xu-Ye Zhao ${ }^{1,2 \&}$, Yong Cui ${ }^{3 \&}$, Shu-Fang Jiang ${ }^{1}$, Ke-Jun Liu ${ }^{1}$, Hai-Qiong Han ${ }^{2}$, \\ Xiao-Su Liu², Yali Li ${ }^{1 *}$
}

\begin{abstract}
Our aims were to evaluate the clinical performance of human telomerase RNA gene component (hTERC gene) amplification assay with high-risk human papillomavirus (HR-HPV) DNA test of Hybrid Capture 2 DNA test (HC2), for the detection of high grade cervical precancerous lesions and cancer (CIN 2+). In addition, the association shown between hTERC gene amplification and HPV DNA test positive in women with and without cervical neoplasia was assessed. There were 92 women who underwent cytology, HR-HPV DNA test, hTERC gene amplification test, colposcopy and biopsy. We compared the clinical performance of hTERC gene test along with HR-HPV DNA test of women with colposcopy and routine screening. The samples were histologyconfirmed high-grade cervical intraepithelial neoplasia (CIN 2) or worse (CIN2+) as the positive criterion. The test of hTERC gene showed the hTERC gene amplification positivity increased with the severity of histological abnormality and cytological abnormality. The test of hTERC gene showed higher specificity than HR-HPV DNA test for high-grade lesions $(84.4 \%$ versus $50 \%)$ and also higher positive predictive value $(\mathbf{9 0 . 4 \%}$ versus $\mathbf{7 6 . 5 \%})$. Our results predicted that hTERC gene amplification demonstrated more specific performance for predicting the risk of progression and offer a strong potential as a tool for triage in cervical cancer screening, with the limited sensitive as HR-HPV DNA test.
\end{abstract}

Keywords: Cervical intraepithelial neoplasias - cervical cancer - human telomerase RNA gene component

Asian Pac J Cancer Prev, 16 (2), 693-697

\section{Introduction}

Cervical cancer is one of the most common gynecological cancer, the third most commonly diagnosed cancer among women and the fourth leading cause of cancer related mortality among women (Jemal et al., 2011), about 29 million people worldwide die of cervical cancer each year, with 3 million people in our country alone, and tend to younger (Baussano et al., 2010). Cervical cancer screening in most countries has worked effectively and reduced the mortality rate remarkably with the using of cytological examination of cervical smears. However, cervical cytology is known to display only a modest sensitivity for cervical precancerous lesion or cancer.

Numerous studies had shown that Infection with high-risk human papillomavirus (HR-HPV) has been recognized as the necessary cause of cervical cancer (Trope et al., 2009). However, most of the HPV infections are asymptomatic and transient, and more than $90 \%$ of new infections will be resolved within 2 years (Moscicki et al., 2006). As such persistent infection with highrisk types is a significant risk factor which initiates the oncogenic process and mediated by up-regulation of E6/ E7 oncological proteins. Thus overexpression of these oncological proteins is associated with an increased risk of lesion progression (Trope et al., 2009). hTERT activation by HPV E6 proteins is uniquely associated with oncogenic types (Van Doorslaer and Burk, 2012). AKT1 loss associated with episomal HPV16 may have positive prognostic implications in vulval intraepithelial neoplasia because the expression of E7 reflects tumour progression (Ekeowa-Anderson et al., 2012). It was reported that HPV genotypes and associated cervical cytological abnormalities in women from the Pearl River Delta region in China (Jing et al., 2014).

Recent research shows that in cervical intraepithelial neoplasias's (CIN's) progression to invasive cervical cancer involves an increase in copy number of the long arm of chromosome 3 (3q) (Kirchhoff et al., 2001). Gain of $3 q$ in cervical cancer has been mapped to $3 q 26-27$, a region which contains human telomerase RNA gene component (hTERC gene) that encodes the template of telomerase RNA (Wilting et al., 2013). Telomerase activation maybe a relatively early event in cervical carcinogenesis, so telomerase activity and expression of its components

${ }^{1}$ Department of Gynecology and Obstetrics, Chinese PLA General Hospital \& Chinese PLA Medical School, ${ }^{2}$ Department of Gynecology, Shanxi Tumor Hospital, Shanxi, ${ }^{3}$ Department of Pathophysiology, Binzhou Medical University, Shandong, China ${ }^{\star}$ Equal contributors*For correspondence: liyalisci@126.com 
may represent valuable biomarkers for the diagnosis and prognosis of patients with cervical neoplasia (Zheng et al., 2000). It has been reported that genomic amplification of the hTERC associated with human papillomavirus was related to the progression of uterine cervical dysplasia to invasive cancer (Liu et al., 2012). Therefore, it is very significance of human telomerase gene amplification detection for cervical cancer screening (Chen et al., 2012; Wang et al., 2013).

The aim of this study was to evaluate the clinical performance of hTERC gene amplification assay with HR-HPV DNA test, for the detection of high grade cervical precancerous lesions and cancer (CIN 2+). And to study the association showed between hTERC gene amplification and HR-HPV DNA positive with the severity of the cervical lesion.

\section{Materials and Methods}

\section{Clinical specimens}

This study is a cross-sectional study using the hospital based analytic design. Participants were recruited consecutively among those who visited Chinese People's Liberation Army General Hospital for a cervical smear in Beijing from March 2011 to August 2012, the number of patients was 92 (median age 42 years, range 24-65 years). Exclusion criteria included a history of treatment for cervical lesions in the previous 1 year or pregnant woman or hysterectomy or any prior type of cancer. All enrolled patients have gone through liquid-based Pap test, HPV DNA test by Hybrid Capture 2 (HC2), and hTERC amplification by Fluorescence in situ hybridization (FISH) detection. The research protocol was approved by the institutional Ethics Committee of Chinese People's Liberation Army General Hospital. All the participants signed an individual informed consent after receiving a detailed explanation of the research. And all the data were processed respecting the confidentiality of the participants. Study design and population.

\section{hTERC amplification test performed by FISH detection}

FISH was performed by an expert blinded to the cytologic and histologic findings, and the procedures were briefly as follows. Briefly, slides were washed in dimethylbenzene and alcohol, and dehydrated in $70 \%, 80 \%$ and $100 \%$ alcohol. In darkness, the labeled oligonucleotide probes were carried out according to manufacturer's instructions. And then, slides were washed in 4X SSCT three times, and 0.1X SSC three times before mounting in DAPI. Finally, Fluorescent microscopy was performed with fluorescent microscope.

\section{HPV DNA testing by $H C 2$}

Testing for HR-HPV DNA was performed principally by using the Hybrid Capture 2 test, a signal amplification test based on the hybridization of 13 high-risk oncogenic types $(16,18,31,33,35,39,45,51,52,56,58,59$, and 68) RNA probe cocktail with the targeted DNA, and the DNA-RNA hybrids can be captured and detected by chemiluminescent. This test was performed with $4 \mathrm{ml}$ of PreservCyt samples according to the manufacturer's instructions. The positive cut off (CO) value was considered the mean of the positive control samples, the results were considered positive when the ratio between the relative-light units of the sample (RLU) and the positive $\mathrm{CO}(\mathrm{RLU} / \mathrm{CO})$ was 1 or greater.

\section{Cytological diagnosis criteria}

Cervical samples were evaluated for cervical cytology in the gynecological Laboratory of Chinese People's Liberation Army General Hospital by two academic cytopathologists blinded to case-control status. Slides were prepared using a liquid-based cytology method, the ThinPrep system (Cytyc Corporation, MA, USA). Cytological classifications of disease grade were made in conformity to Bethesda2001 criteria (Anttila et al., 2010). A diagnosis was assigned to each case as having negative for intraepithelial lesion or malignancy (NILM) or having an epithelial cell abnormality such as atypical squamous cells of undetermined significance (ASC-US), atypical squamous cells, cannot exclude high-grade squamous intraepithelial lesion (ASC-H), low-grade squamous intraepithelial lesion (LSIL), high-grade squamous intraepithelial lesion (HSIL), squamous cell carcinoma (SCC) and atypical glandular cells (AGC) (Solomon et al., 2002).

\section{Histological diagnosis criteria}

Cervical biopsies were taken only from those women with abnormal colposcopy, and this was performed at the time of cervical cytology specimen collection. Colposcopy was conducted by participating obstetrics and gynecology (OB/GYN) specialists from Chinese People's Liberation Army General Hospital blinded to case-control status. Cervical biopsy results were obtained and read by two or more pathologists from Chinese People's Liberation Army General Hospital blinded to case-control status. All slides were diagnosed according to the current World Health Organization.

\section{Statistical analysis}

All statistical analyses were performed using SPSS 17.0 statistical package for Windows. Pearson's $\chi^{2}$ test was used to evaluate the significance of differences between designated groups. $\mathrm{P}<0.05$ was considered significant. Sensitivity, specificity, and predictive values were calculated using the conventional contingency tables, and $95 \%$ confidence intervals (95\% CIs) were computed using exact binomial methods.

\section{Results}

Cytologic or histologic diagnosis and positivity rate for hTERC gene amplification

All enrolled patients, overall 92 patients (median age 42 years, range 24-65 years) had HR-HPV DNA test and liquid-based cytology (LBC) on the same sample. According to the cytological indexes, 52 out of 92 samples $(56.5 \%)$ were hTERC gene amplification test positive. HTERC gene amplification test positivity rates rose from $22.7 \%(5 / 22)$ for NILM (negative for intraepithelial lesion or malignancy), $44 \%$ (11/25) for ASCUS (atypical 
squamous cells of undetermined significance) and $60 \%$ (12/20) for LSIL (low-grade squamous intraepithelial lesion), 96\% (24/25) for ASC-H+ (atypical squamous cells, can't exclude high-grade squamous intraepithelial lesion). Histology revealed that, of the 92 specimens,

Table 1. Cytological or Histological Diagnosis and Positivity Rate for hTERC Gene Amplification

\begin{tabular}{lrr}
\hline Follow-up diagnosis & $\begin{array}{c}\text { No. }(\%) \text { of } \\
\text { women }\end{array}$ & $\begin{array}{r}\text { No. of positive } \\
\text { for hTERC }(\%)\end{array}$ \\
\hline Cytology result & $22(23.9)$ & $5(22.7 \%)$ \\
$\quad$ Normal & $25(27.2)$ & $11(44 \%)$ \\
ASCUS & $20(21.7)$ & $12(60 \%)$ \\
LSIL & $25(27.2)$ & $24(96 \%)$ \\
ASC-H+a & $92(100)$ & $52(56.5 \%)$ \\
Total & $10(10.9)$ & $1(10 \%)$ \\
Histology result & $22(23.9)$ & $4(18.2 \%)$ \\
normal & $24(26.1)$ & $14(58.3 \%)$ \\
CIN1 & $15(16.3 \%)$ & $12(80 \%)$ \\
CIN2 & $21(22.8)$ & $21(100 \%)$ \\
CIN3 & $92(100 \%)$ & \\
Carcinoma & \\
Total & & \\
*aASC-H+, include ASC-H, HSIL, SCC and AGC
\end{tabular}

Table 2. Comparative Performance of hTERC Gene Amplification Test and HR-HPV DNA Assays ${ }^{\mathrm{a}}$

\begin{tabular}{lccc}
\hline HR-HPV DNA Number & \multicolumn{2}{c}{$\begin{array}{c}\text { No. of hTERC gene } \\
\text { amplification results }\end{array}$} \\
\cline { 2 - 3 } & Positive cases & The positive rate, $\%$ \\
\hline Negative & 24 & 7 & 29.2 \\
Positive & 68 & 45 & 66.2 \\
Total & 92 & 52 & 56.5 \\
\hline
\end{tabular}

Table 3. Correlation of hTERC Gene Amplification with Histological Diagnoses

\begin{tabular}{lccc}
\hline HTERC & \multicolumn{3}{c}{ No. of histological diagnoses } \\
\cline { 2 - 4 } Gene amplification & CIN2 $^{\text {+a }}$ & CIN1 $^{-b}$ & Total \\
Positive & 47 & 5 & 52 \\
Negative & 13 & 27 & 40 \\
Total & 60 & 32 & 92 \\
\hline
\end{tabular}

*aCIN2+, include CIN2, CIN3, SCC and adenocarcinoma; ${ }^{\mathrm{b}} \mathrm{CIN} 1-$, include NILM, CIN1, and BNA (borderline nuclear abnormalities)

Table 4. Correlation of HR-HPV DNA with Histological Diagnoses

\begin{tabular}{lccc}
\hline HR-HPV DNA & \multicolumn{3}{c}{ No. of histological diagnoses } \\
\cline { 2 - 4 } Gene amplification & CIN2 & CIN1 & Total \\
\hline Positive & 52 & 16 & 68 \\
Negative & 8 & 16 & 24 \\
Total & 60 & 32 & 92 \\
\hline
\end{tabular}

*acIN2+, include CIN2, CIN3, SCC and adenocarcinoma; ${ }^{\mathrm{b}} \mathrm{CIN} 1-$, include NILM, CIN1, and BNA (borderline nuclear abnormalities)
$10(10.9 \%)$ were normal, $22(23.9 \%)$ were classified as CIN1, $24(26.1 \%)$ as CIN2, $15(16.3 \%)$ as CIN3, and 21 $(22.8 \%$ ) as cervical carcinoma (Table 1$)$. The positive rate of hTERC gene amplification rose from $10 \%(1 / 10)$ in normal, $18.2 \%$ (4/22) in CIN 1 to $58.3 \%$ (14/24) in CIN2, $80 \%(12 / 15)$ in CIN3 and $100 \%(21 / 21)$ in carcinoma (squamous cell carcinoma and adenocarcinoma) (Table 1).

Comparative performance of hTERC gene amplification test and HR-HPV DNA assay

To better investigate the performance of hTERC gene amplification test and HR-HPV DNA assay, the data concerning the group of 92 patients which was detected by these two tests was analyzed. The concordance between the two tests was $67.4 \%$ (62/92), with 45 cases double positive and 17 cases double negative with both tests. Accordingly, there was discordance in 30 cases $32.6 \%$ (30 / 92). hTERC gene amplification test was positive in 7 cases, which were negative with HR-HPV DNA test, while 23 cases were positive with HR-HPV DNA test and negative with hTERC gene amplification test. The positivity rates of hTERC gene amplification test and HRHPV DNA assays were 56.5\% (52/92) and 73.9\% (68/92), respectively. The positivity rate $29.2 \%$ (7/24) for hTERC gene amplification was higher in the HR-HPV infected patients than $66.2 \%(45 / 68)$ in HR-HPV negative patients, there was significant differences $(\mathrm{P}<0.05)$, suggesting the infection of HR-HPV DNA is closely related to the positivity of the hTERC gene amplification.

Correlation of hTERC gene amplification test and HRHPV DNA with CIN 2+

The detection of hTERC gene amplification test in cervical specimens and HR-HPV DNA were analyzed in relation to histology status. The prevalence of CIN 2+ was $65.2 \%$ (60/92). The specificity was significantly higher for hTERC gene amplification test 84.4\% (27/32) (95\% CI, $68.25,93.14)$ than that of HR-HPV DNA test $50 \%(16 / 32)$ (95\% CI, 33.6, 66.4) (Tables 3, 4 and 5).

\section{Discussion}

Cervical cancer has become a global health problem, the use of HR-HPV DNA tests in primary screening have been shown in several studies to be more sensitive than conventional cytology in detecting cervical cancer and severe precancerous lesions and may serve to prolong the screening interval (Anttila et al., 2010). But the specificities of HR-HPV DNA tests for identification of cervical neoplasia are lower than those for cytology, especially among younger women (Szarewski et al., 2008). Recent studies showed that the detection of the hTERC gene amplification and abnormal cervical screening of

Table 5. Comparison of hTERC Gene Amplification Test and HR-HPV DNA for Detection CIN2+ in Referral Population by Histological Grade ${ }^{a}$

\begin{tabular}{lcccc}
\hline Tests & Sensitivity, 95\%CI & Specitivity, 95\%CI & PPV, 95\%CI & NPV, 95\%CI \\
\hline hTERC gene amplification & $78.3(66.4,86.9)$ & $84.4(68.25,93.14)$ & $90.4(79.4,95.8)$ & $67.5(52.0,79.9)$ \\
HR-HPV DNA & $86.7(75.8,93.1)$ & $50(33.6,66.4)$ & $76.5(65.1,85.05)$ & $66.7(46.7,82.0)$ \\
\hline${ }^{* a} \mathrm{n}=92$ & & & &
\end{tabular}


high-risk lesions has clinical significance. Monitoring the development of cervical lesions has important clinical value.

In the present study, hTERC gene amplification assay showed that the hTERC gene amplification positivity increased with severity of histological abnormality and ranged from $15.6 \%$ (5/32) for CIN 1- to $66.7 \%$ (26/39) in CIN2-3 and $100 \%$ (37/40) in carcinoma. Women with CIN2+ were more likely to test positive for hTERC gene amplification than those with CIN1- (78.3\% versus 15.6\%; $P<0.01)$. The overall positivity rate of the hTERC gene amplification assay was $56.5 \%$ (Table 1). Similar results are presented here, many studies have confirmed hTERC gene from chronic cervicitis and CIN to cervical lesions, its amplification yields a positive rate and was positively correlated with severity. High-level cervical lesions often present hTERC gene amplification (Costa et al., 2009; Sui et al., 2009; Chen et al., 2011).

In the present study, hTERC assay showed that the positivity of hTERC gene amplification also increased with the severity of cytological abnormality, ranging from $22.7 \%$ (5/22) for NILM, $51 \%$ (23/45) for ASCUS and LSIL, 96\% (24/25) for ASC-H+. That is consistent with the previous studies, Heselmeyer-Haddad and others found three-color FISH probes for cervical smears and detected 3q26 hTERC genes. This gene amplification was found to aggravate hTERC with cytological lesions increased (Pett et al., 2006). Caraway NP et al used the fluorescence in situ hybridization analyzed cervical cytology samples showed cervical intraepithelial neoplasia had the same degree of progress; and the abnormal hTERC gene amplification rate was gradually increased (Caraway et al., 2008). Zhang proposed that the hTERC gene FISH assay can be accurately distinguished between low-grade and high-grade cervical intraepithelial neoplasia and that this can be used for secondary screening of high-risk patients (Zhang et al., 2009).

In the present study, hTERC gene amplification test showed the higher specificity than HC2 test $(84.4 \%$ verse $50 \%$ ). Current research given that referring all HR-HPV DNA-positive women to colposcopy will result in over diagnosis, overtreatment, and high costs. The use of hTERC gene amplification test will significantly reduce the number of women without CIN2+ referred to colposcopy or requiring further assessment. There may be merit for using hTERC gene amplification test in triage or as an add-on to HPV DNA testing.

In the present study, the comparative performance of hTERC gene amplification test and HC2 showed a concordance of $67.4 \%$. In terms of clinical performance, this is consistent with many previous studies, the hTERC gene amplification with the HPV infection and host genes in the process of integration has played a key role In cervical precancerous lesions (Hopman et al., 2006). HRHPV infection may undergo abnormal gene amplification as early events. hTERC increases telomerase activity. The HPV oncogenic mechanism may be an important part of host cells that are infected with a HPV telomerase gene amplification. It leads to lesions pathological changes and this genetic alteration becomes more and more serious as the disease progresses (Oikonomou et al., 2006).
This study was limited by the lack of follow-up data. A prospective follow-up was planned for further increase the sample size to reduce this bias.

In conclusions, our results suggest that hTERC gene amplification test demonstrated more specific performance for detecting CIN2+ with the limited sensitive as HR-HPV DNA and have the potential to serve as a more specific test for predicting the risk of progression and offer a strong tool for triage in cervical cancer screening. Further investigations are needed to follow-up in screening setting.

\section{Acknowledgements}

This work was supported by Chinese People's Liberation Army General Hospital.

\section{References}

Anttila A, Kotaniemi-Talonen L, Leinonen M, et al (2010). Rate of cervical cancer, severe intraepithelial neoplasia, and adenocarcinoma in situ in primary HPV DNA screening with cytology triage: randomised study within organised screening programme. $B M J, \mathbf{3 4 0}, 1804$.

Baussano I, Ronco G, Segnan N, et al (2010). HPV-16 infection and cervical cancer: modeling the influence of duration of infection and precancerous lesions. Epidemics, 2, 21-8.

Caraway NP, Khanna A, Dawlett M, et al (2008). Gain of the 3 q26 region in cervicovaginal liquid-based pap preparations is associated with squamous intraepithelial lesions and squamous cell carcinoma. Gynecol Oncol, 110, 37-42.

Chen Q, Wang X, Ru Y, et al (2011). Amplification of the telomerase RNA component gene in the process of human esophageal carcinogenesis. Tohoku J Exp Med, 224, 99-104.

Chen SM, Lin W, Liu X, et al (2012). Significance of human telomerase RNA gene amplification detection for cervical cancer screening. Asian Pac J Cancer Prev, 13, 2063-8.

Costa C, Espinet B, Molina MA, et al (2009). Analysis of gene status in cervical dysplastic lesions and squamous cell carcinoma using tissue microarrays. Histol Histopathol, 24, 821-9.

Ekeowa-Anderson AL, Purdie KJ, Gibbon K, et al (2012). AKT1 loss correlates with episomal HPV 16 in vulval intraepithelial neoplasia. PLoS One, 7, 38608.

Hopman AH, Theelen W, Hommelberg PP, et al (2006). Genomic integration of oncogenic HPV and gain of the human telomerase gene TERC at $3 \mathrm{q} 26$ are strongly associated events in the progression of uterine cervical dysplasia to invasive cancer. $J$ Pathol, 210, 412-9.

Jemal A, Bray F, Center MM, et al (2011). Global cancer statistics. CA Cancer J Clin, 61, 69-90.

Jing L, Zhong X, Huang W, et al (2014). HPV genotypes and associated cervical cytological abnormalities in women from the Pearl River Delta region of Guangdong province, China: a cross-sectional study. BMC Infect Dis, 14, 388 .

Kirchhoff M, Rose H, Petersen BL, et al (2001). Comparative genomic hybridization reveals non-random chromosomal aberrations in early preinvasive cervical lesions. Cancer Genet Cytogenet, 129, 47-51.

Liu H, Liu S, Wang H, et al (2012). Genomic amplification of the human telomerase gene (hTERC) associated with human papillomavirus is related to the progression of uterine cervical dysplasia to invasive cancer. Diagn Pathol, 7, 147.

Moscicki AB, Schiffman M, Kjaer S, et al (2006). Chapter 5: Updating the natural history of HPV and anogenital cancer. Vaccine, 24, 42-51.

Oikonomou P, Mademtzis I, Messinis I, et al (2006). Quantitative 
determination of human telomerase reverse transcriptase messenger RNA expression in premalignant cervical lesions and correlation with human papillomavirus load. Hum Pathol, 37, 135-42.

Pett MR, Herdman MT, Palmer RD, et al (2006). Selection of cervical keratinocytes containing integrated HPV16 associates with episome loss and an endogenous antiviral response. Proc Natl Acad Sci U S A, 103, 3822-7.

Sui W, Ou M, Dai Y, et al (2009). Gain of the human telomerase RNA gene TERC at $3 \mathrm{q} 26$ is strongly associated with cervical intraepithelial neoplasia and carcinoma. Int J Gynecol Cancer, 19, 1303-6.

Szarewski A, Ambroisine L, Cadman L, et al (2008). Comparison of predictors for high-grade cervical intraepithelial neoplasia in women with abnormal smears. Cancer Epidemiol Biomarkers Prev, 17, 3033-42.

Trope A, Sjoborg K, Eskild A, et al (2009). Performance of human papillomavirus DNA and mRNA testing strategies for women with and without cervical neoplasia. J Clin Microbiol, 47, 2458-64.

Van Doorslaer K, Burk RD (2012). Association between hTERT activation by HPV E6 proteins and oncogenic risk. Virology, 433, 216-9.

Wang YF, Wang XS, Gao SG, et al (2013). Clinical significance of combined detection of human papilloma virus infection and human telomerase RNA component gene amplification in patients with squamous cell carcinoma of the esophagus in northern China. Eur J Med Res, 18, 11.

Wilting SM, Snijders PJ, Verlaat W, et al (2013). Altered microRNA expression associated with chromosomal changes contributes to cervical carcinogenesis. Oncogene, 32, 106-16.

Zhang Y, Wang X, Ma L, et al (2009). Clinical significance of hTERC gene amplification detection by FISH in the screening of cervical lesions. J Huazhong Univ Sci Technolog Med Sci, 29, 368-71.

Zheng PS, Iwasaka T, Zhang ZM, et al (2000). Telomerase activity in Papanicolaou smear-negative exfoliated cervical cells and its association with lesions and oncogenic human papillomaviruses. Gynecol Oncol, 77, 394-8. 